\title{
Multigate Buckled Self-Aligned Dual Si Nanowire MOSFETs on Bulk Si for High Electron Mobility
}

\author{
M. Najmzadeh, Student Member, IEEE, Y. Tsuchiya, D. Bouvet, W. Grabinski, Senior Member, IEEE, \\ and A. M. Ionescu, Senior Member, IEEE
}

\begin{abstract}
In this paper, we report for the first time making multi-gate buckled self-aligned dual $\mathrm{Si}$ nanowires including two sub-100 nm cross-sectional cores on bulk Si substrate using optical lithography, hard mask/spacer technology, and local oxidation. $\approx 0.8$ GPa uniaxial tensile stress was measured on the buckled dual nanowires using micro-Raman spectroscopy. The buckled multigate dual Si nanowires show excellent electrical characteristics, e.g., $62 \mathrm{mV} /$ decade and $42 \%$ low-field electron mobility enhancement due to uniaxial tensile stress in comparison to the non-strained device, all at $V_{D S}=50 \mathrm{mV}$ and $293 \mathrm{~K}$.
\end{abstract}

Index Terms-Local oxidation, local stressor, micro-Raman spectroscopy, MOSFET, multi-gate, Si nanowire, strain engineering, uniaxial tensile stress.

\section{INTRODUCTION}

M ULTI-GATE devices such as gate-all-around $\mathrm{Si}$ nanowires are promising candidates during aggressive CMOS downscaling, due to having optimized subthreshold slope, immunity against short-channel effect and optimized power consumption. For further performance enhancement while downscaling especially for the 90-nm technology nodes and beyond, CMOS boosters, e.g., stressors have been integrated in the CMOS process flows. According to [1]-[3], Si nanowires show excellent mechanical properties versus bulk $\mathrm{Si}$ in terms of yield and fracture strengths. On the other hand, due to the freedom on buckling in the suspended Si nanowires, a significant level of stress in the Si channel can be obtained [4], [5]. Therefore, the Si nanowires can be counted as the best innovation platforms for various strain engineering-based CMOS booster applications, e.g., significant stress level modulation in the channel [5] to boost the carrier mobility in the classical MOSFETs and stress profile engineering along the channel [6], [7] for band-to-band tunneling current boost in tunnel FETs. Including stress in the suspended channels is also a technology challenge and until now only a few methods are reported: strained

Manuscript received January 9, 2012; accepted June 3,2012. Date of publication June 20, 2012; date of current version September 1, 2012. This project is supported by Swiss National Science Foundation (SNSF). The review of this paper was arranged by Associate Editor C. Zhou.

M. Najmzadeh, D. Bouvet, W. Grabinski, and A. M. Ionescu are with the Nanoelectronic Devices Laboratory (Nanolab), Swiss Federal Institute of Technology, 1015 Lausanne, Switzerland (e-mail: mohammad.najmzadeh@epfl.ch; didier.bouvet@epfl.ch; wladyslaw.grabinski@epfl.ch; adrian.ionescu@epfl.ch).

Y. Tsuchiya is with the Nano Group, School of Electronics and Computer Science, University of Southampton, Highfield Campus, Southampton, SO17 1BJ, U.K. (e-mail: yt2@ecs.soton.ac.uk).

Color versions of one or more of the figures in this paper are available online at http://ieeexplore.ieee.org.

Digital Object Identifier 10.1109/TNANO.2012.2205401 substrate [8], metal gate strain [4], local oxidation [9], SiGe S/D [10], diamond-like carbon (DLC) [11], and local oxidation and metal gate strain [5], [12]-[14].

Parallel to the CMOS boosters, making a 3-D stack of multigate devices using various techniques, e.g., dual SOI wafer [15], epitaxial Si-SiGe stacks [16], [17], and stress-limited oxidation [18] can enhance further the current density. Among the 3-D integration techniques, stress-limited oxidation is the simplest way used previously to make dual Si nanowires based on a vertical fin on silicon on insulator (SOI) [18], and vertical stack of Si nanowires from a preshaped vertical fin using scalloping on bulk Si [19]. A well-controlled stress-limited oxidation can even cause keeping a thin $\mathrm{Si}$ bridge between the vertical $\mathrm{Si}$ nanowire cores, leading to a significant improve in drive current [20], while keeping the nanowires in a more self-aligned manner and helping to suppress the sticking issue of the dense array of parallel nanowires due to the possible buckling in the case of, e.g., using local stressors and, therefore, providing more freedom to make a more dense array structure from the top-down Si nanowire platforms.

In this paper, we are reporting the fabrication and characterization of strained self-aligned dual Si nanowires on bulk Si substrate using $0.8-\mu \mathrm{m}$ optical lithography and hard mask/spacer technology for the first time. The stress value is measured in the buckled Si nanowires by micro-Raman spectroscopy and finally, the low-field electron mobility boost due to stress is reported.

\section{FABRICATION PROCESS}

The process flow to fabricate self-aligned dual Si nanowires from bulk Si substrate is demonstrated in Fig. 1. As the first step, the $100 \mathrm{~mm}\left(\begin{array}{lll}1 & 0 & 0\end{array}\right)$ p-type $(0.1-0.5 \Omega \cdot \mathrm{cm})$ bulk prime $\mathrm{Si}$ wafers were oxidized using wet oxidation at $1050{ }^{\circ} \mathrm{C}$ to grow $500 \mathrm{~nm}$ of $\mathrm{SiO}_{2}$ including - $310 \mathrm{MPa}$ residual thin-film biaxial compressive stress. Afterward, DWL200 was used to write the active resist pattern (S/D and wires) directly on the wafer using $0.8-\mu \mathrm{m}$ resolution optical lithography. The length and the width of the wire masks were varying from 2.0 to $20.0 \mu \mathrm{m}$ and 0.8 to $1.8 \mu \mathrm{m}$, respectively. To transfer the active layer to the wafer, the $\mathrm{SiO}_{2}$ hard mask and $\mathrm{Si}$ were etched anisotropically using fluorine-based chemistry to make $\approx 1.1 \mu \mathrm{m}$ high ribs. A 5 hour sacrificial wet oxidation at $850{ }^{\circ} \mathrm{C}$ was done to narrow the $\mathrm{Si}$ ribs from the two sides, smooth the Si side walls, and simultaneously grow an oxide spacer layer. A short fluorine-based dry anisotropic $\mathrm{SiO}_{2}$ etching step was performed to remove the grown $\mathrm{SiO}_{2}$ on the substrate. The higher oxidation rate for the $\mathrm{Si}$ side walls in comparison to the (1 00$) \mathrm{Si}$ surface leading to grow a thicker oxide on the side walls while due to the slightly 

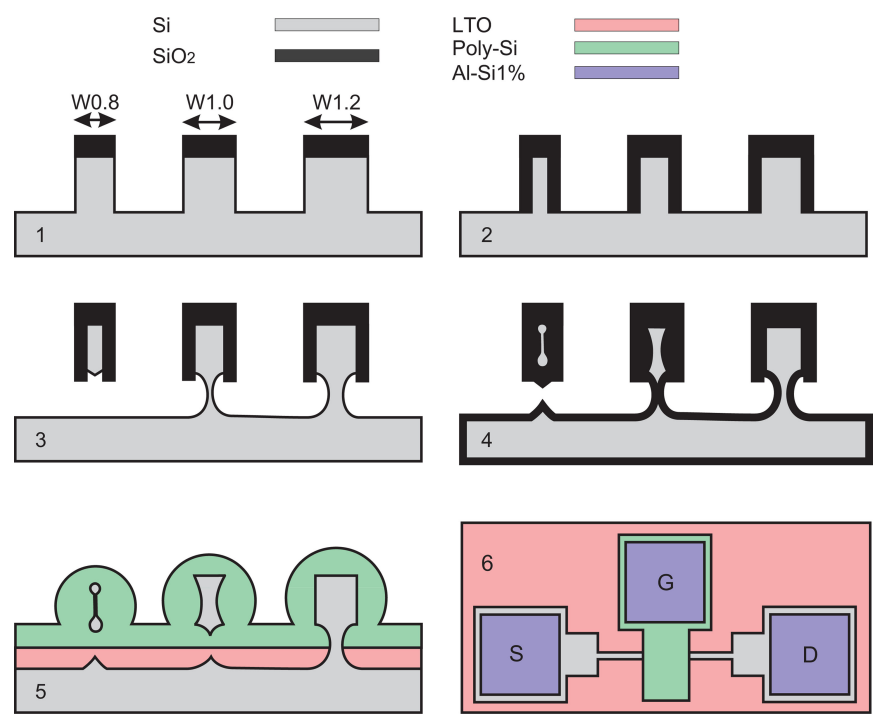

Fig. 1. Process flow to obtain multigate Si nanowire MOSFETs on bulk. W0.8 corresponds to the initial $0.8 \mu \mathrm{m}$ mask nanowire width.

slanted side walls, the final $\mathrm{SiO}_{2}$ spacer thickness right after the $\mathrm{SiO}_{2}$ etching step is estimated to be $\leq 50 \mathrm{~nm}$. Afterward, a fluorine-based isotropic Si etching was done to suspend the narrowest ribs $(0.8 \mu \mathrm{m}$ initial mask NW width, called W0.8 in this paper) from the substrate, while the rest of the Si ribs were still connected to the substrate.

A 4 hour wet oxidation at $850{ }^{\circ} \mathrm{C}\left(8.3 \mathrm{~L} / \mathrm{min} \mathrm{O}_{2}, 16.0 \mathrm{~L} / \mathrm{min}\right.$ $\mathrm{H}_{2}$ ) was done to nominally consume $120 \mathrm{~nm}$ of $\left(\begin{array}{lll}1 & 0 & 0\end{array}\right) \mathrm{Si}$ to reduce the nanowire dimensions, consume the remained $\mathrm{Si}$ between the wider nanowires (W1.0) and substrate to suspend the structures. The oxidation temperature was chosen to be below the glass transition temperature of $\mathrm{SiO}_{2}, 960{ }^{\circ} \mathrm{C}$, to avoid relaxation of stress in the $\mathrm{SiO}_{2}$ thin films to be able to accumulate mechanical potential energy in the nanowires during oxidation [21], [22]. The higher oxidation rate for the Si side walls in comparison to the top (1 00 ) Si surface as well as builtin stress in the growing oxide layer during the oxidation step yields to obtain two Si cores with rounded corners connected with a sub-10 $\mathrm{nm}$ thick Si bridge after oxidation of the narrowest Si ribs (W0.8).

Stripping the $\mathrm{SiO}_{2}$ hard mask/spacer and the grown oxide was done using wet buffered hydrofluoric (BHF) etching (volume ratio of 7:1 of $\mathrm{NH}_{4} \mathrm{~F} 40 \%$ and $\mathrm{HF} 50 \%$ ). Fig. 2 represents an array of Si nanowires including two sub-100 nm cross-sectional self-aligned Si cores connected with a thin Si bridge right after the stripping step. The buckling in the nanowires after the stripping step is due to releasing the stored mechanical potential energy in the nanowires during the oxidation step [21], [22], a clear sign of uniaxial tensile stress in the nanowire.

An isolation step including low-temperature oxide (LTO) deposition, CMP, and isotropic LTO etching using BHF was done to isolate the suspended nanowires from bulk. The gate stack step includes RCA, dry oxidation to grow $15 \mathrm{~nm}$ of gate oxide at $850{ }^{\circ} \mathrm{C}$ and finally $230 \mathrm{~nm}$ of LPCVD in-situ doped $\mathrm{N}^{+}$polySi (phosphorous: $2 \times 10^{20} \mathrm{~cm}^{-3}$ ) at $480{ }^{\circ} \mathrm{C}$. The thin Si bridge during the gate stack step is being consumed and finally two

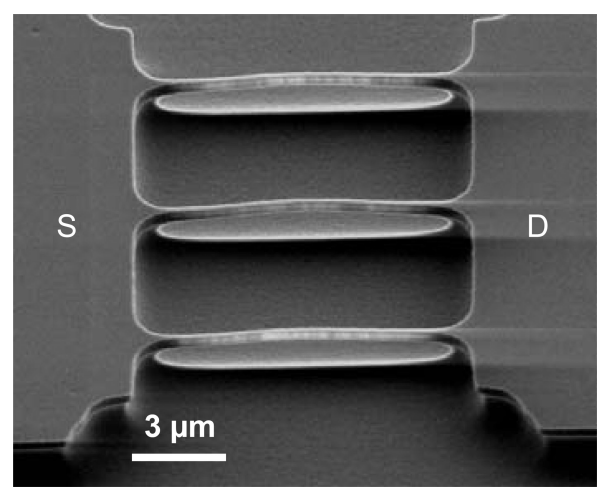

Fig. 2. Tilted-view SEM micrograph of an array of $10 \mu \mathrm{m}$ long buckled dual $\mathrm{Si}$ nanowires on bulk $\mathrm{Si}$ right after the $\mathrm{SiO}_{2}$ stripping step, representing the reproducibility of the process flow from strain engineering and dual Si nanowire formation aspects. The two sub-100 $\mathrm{nm}$ cross-sectional nanowire cores are connected to each other using a thin-Si bridge. The out-of-plane buckling is the signature of uniaxial tensile stress in the nanowire.

separated self-aligned sub-100 nm cross-sectional Si cores can be obtained (see Fig. 3).

To activate the dopants in the poly-Si thin film, a 5 min furnace annealing at $900{ }^{\circ} \mathrm{C}$ was done. The gate pattern was transferred to the wafer using optical lithography and fluorinebased isotropic poly-Si etching. Stripping the resist layer, S/D phosphorous ion implantation $\left(2 \times 10^{15} \mathrm{~cm}^{-2}, 30 \mathrm{keV}\right)$ and annealing to activate the dopants, metallization, and sintering $\left(450{ }^{\circ} \mathrm{C}, 30 \mathrm{~min}\right)$ were the further steps.

\section{StRAin ANALYSIS IN THE BUCKLED Si NANOWIRES}

\section{A. Stress Measurement on the Buckled Dual Si Nanowires by Micro-Raman Spectroscopy}

Micro-Raman spectroscopy was employed for measuring stress on a finalized dual Si nanowire MOSFET at 293 K. A Renishaw inVia spectrometer setup is used with a green laser of $532 \mathrm{~nm}$ in wavelength. As the penetration depth of the laser in $\mathrm{Si}(\approx 1 \mu \mathrm{m})$ is longer than the thickness of the Si nanowire $(<500 \mathrm{~nm})$, both Raman spectra of the strained Si nanowire and the relaxed $\mathrm{Si}$ underneath are observed, while the laser beam is focused on the nanowire. The line scan with the step of 0.5 $\mu \mathrm{m}$ was done across the naked part of the Si nanowire between S/D pad and gate pattern (see Fig. 4) as the measurement on the $\mathrm{Si}$ channel via the poly-Si gate stack is challenging due to the broad spectra of poly-Si around the crystalline Si spectra.

A spectrum shown in Fig. 5 was taken at the 10th from the top out of 16 points along the scan axis. A considerable satellite peak is only observed at this point while just a single peak originated from relaxed bulk $\mathrm{Si}$ at $520.6 \mathrm{~cm}^{-3}$ is observed in the others except for a very subtle shoulder at the 9 th and 11 th points. The result suggests that the buckled nanowire was spotted on in the measurement at the 10th point. As shown in Fig. 5, the corresponding two peaks are deconvoluted from the spectrum by using Lorentzian peak fitting [23]. 0.465\%/0.785 GPa uniaxial tensile strain/stress in the buckled Si nanowire are estimated by assuming Young's modulus of $169 \mathrm{GPa}$ in $\mathrm{Si}$. 


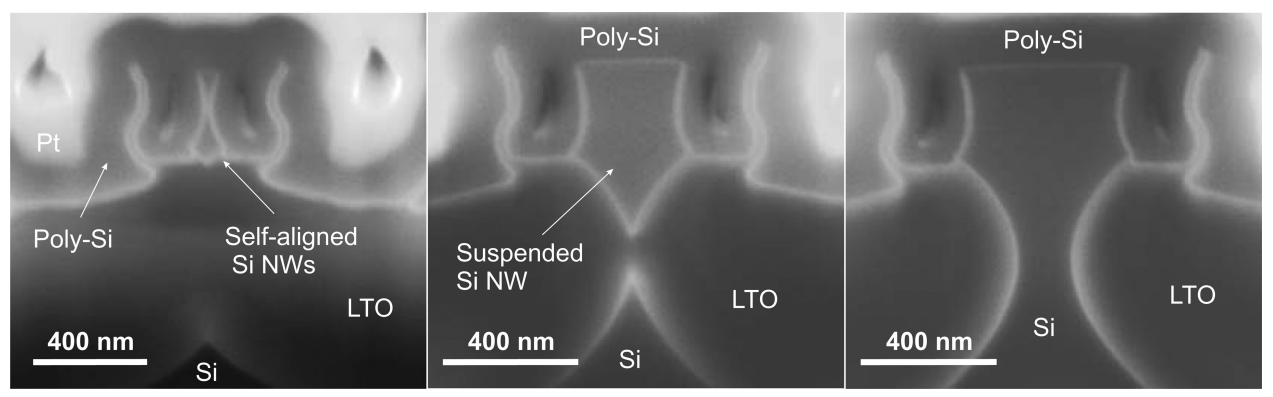

Fig. 3. SEM nanograph from the cross-section of multi-gate MOSFETs on bulk Si: multi-gate suspended dual self-aligned Si nanowires (W0.8, left), multi-gate suspended Si nanowire (W1.0, center) and omega-gate MOSFET (W1.2, right).

\section{B. Process-Based Stress Optimization in the Top-Down Si Nanowire Platform}

Previously in [21] and [22], we reported in-depth stress analysis of local oxidation of the $\mathrm{Si}$ nanowires with a $\mathrm{Si}_{3} \mathrm{~N}_{4} / \mathrm{SiO}_{2}$ tensile hard mask on the top. In this paper, due to using a $\mathrm{SiO}_{2}$ hard mask, grown by wet oxidation, the sign of the stress in the hard mask is reverse because of volume expansion during oxidation. Less restrictions on out-of-plane buckling during the oxidation step due to having a compressive hard mask on top leads to a smaller accumulation of mechanical potential energy during oxidation, and finally a smaller amount of stress in the $\mathrm{Si}$ nanowires is expected in comparison to the $\mathrm{Si}$ nanowires with a tensile hard mask on the top. On the other hand, using a $\mathrm{SiO}_{2}$ hard mask and spacer has the advantage of further rounding the sharp corners of the Si nanowire channel. To improve further the level of uniaxial tensile stress in the Si nanowires, a high-k/metal-gate stack including an intrinsic compressive thin-film stress in the metal-gate layer can be used [4], [5]. As a consequence, to even significantly relax the level of stress to make the Si nanowire platform suitable for the pMOS devices without hole mobility degradation, the thermal oxidation can be performed at temperatures higher than the glass transition temperature of $\mathrm{SiO}_{2}, 960{ }^{\circ} \mathrm{C}$, for viscoelastic stress relaxation in the $\mathrm{SiO}_{2}$ thin films on the wafer and further compressive stress can be induced to the suspended nanowire channel using a high- $\mathrm{k} /$ metal-gate stack including an intrinsic biaxial tensile stress in the metal-gate layer.

\section{ELECTRICAL CHARACTERIZATION AND EXTRACTION OF PARAMETERS}

Electrical characterization was done at $293 \mathrm{~K}$ using a Cascade prober and a HP 4155B Semiconductor Parameter Analyzer. Figs. 6 and 7 show output and input characteristics of the multi-gate dual Si nanowire MOSFET with the shown SEM micrographs in Figs. 3 (left) and 4. The threshold voltage and low-field mobility values were extracted using the $\mathrm{I}_{D} / \sqrt{g_{m}}$ method [24], [25], quasi-independent of series resistance and mobility attenuation factor reporting $\mathrm{V}_{\mathrm{TH}}=-0.061 \mathrm{~V}$ and $\mu_{0}$ $=468 \mathrm{~cm}^{2} / \mathrm{V} \cdot \mathrm{s}$ at $\mathrm{V}_{\mathrm{DS}}=50 \mathrm{mV}$. According to Fig. 7 , the $\mathrm{I}_{\mathrm{on}} / \mathrm{I}_{\text {off }}$ ratio at $\mathrm{V}=2.000 \mathrm{~V}$ can be as high as $\approx 10^{8}$ in the case of tuning the threshold voltage using a metal gate with a mid-gap work function or channel doping modulation. During the extraction of parameters, only the covered faces of the nanowires with the

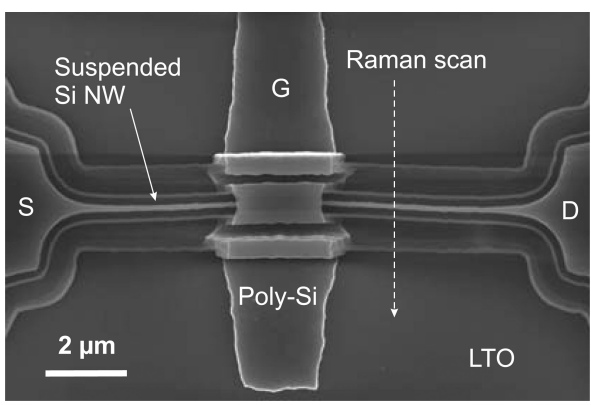

Fig. 4. Top-view SEM micrograph of a multi-gate dual Si nanowire MOSFET on bulk Si. The in-plane nanowire buckling is a sign of uniaxial tensile stress in the channel. The arrow indicates the scan axis and direction of the laser spot in the micro-Raman measurement.

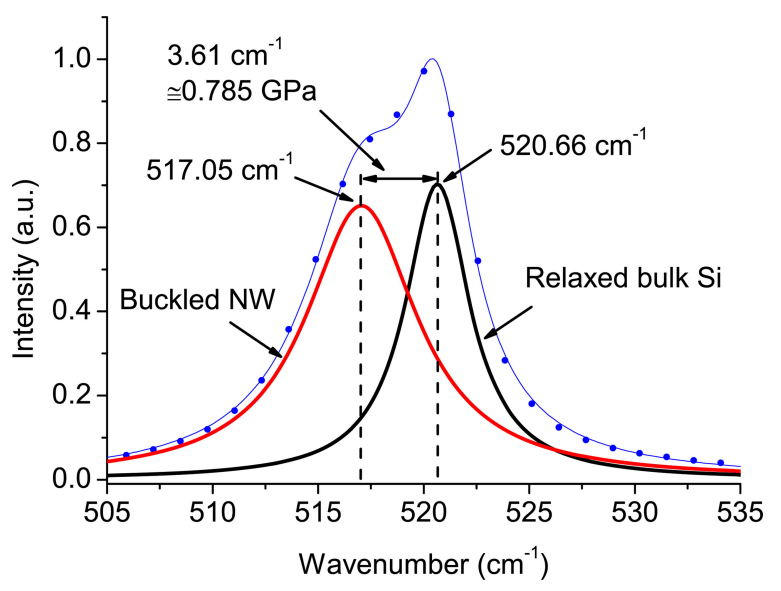

Fig. 5. Micro-Raman spectrum taken on a $12 \mu \mathrm{m}$ long buckled dual $\mathrm{Si}$ nanowire on bulk Si (SEM top-view in Fig. 4). The spectrum is well fitted with two Lorentzian peaks corresponding to the relaxed bulk Si at $520.66 \mathrm{~cm}^{-1}$ and the strained Si nanowire at $517.05 \mathrm{~cm}^{-1}$.

poly-Si/SiO ${ }_{2}$ gate stack in Fig. 3 were considered as the $\mathrm{W}_{\text {eff }}$ of the MOSFETs. Note that the bottom part of the suspended nanowires not covered with the gate stack, mainly due to its challenging isolation step, acts as an independent transistor with a thick gate-oxide layer ( $>200 \mathrm{~nm}$ LTO) and using bulk Si as the back gate. Due to its fairly thick oxide thickness and since $V_{B S}$ is fixed to $0 \mathrm{~V}$ during all the measurements, this independent transistor cannot involve significantly in the conduction.

To compare the results, the wider MOSFETs with the SEM cross-sections in Fig. 3 and with the same gate length in Fig. 4 were also characterized (see Figs. 8 and 9). The omega-gate 


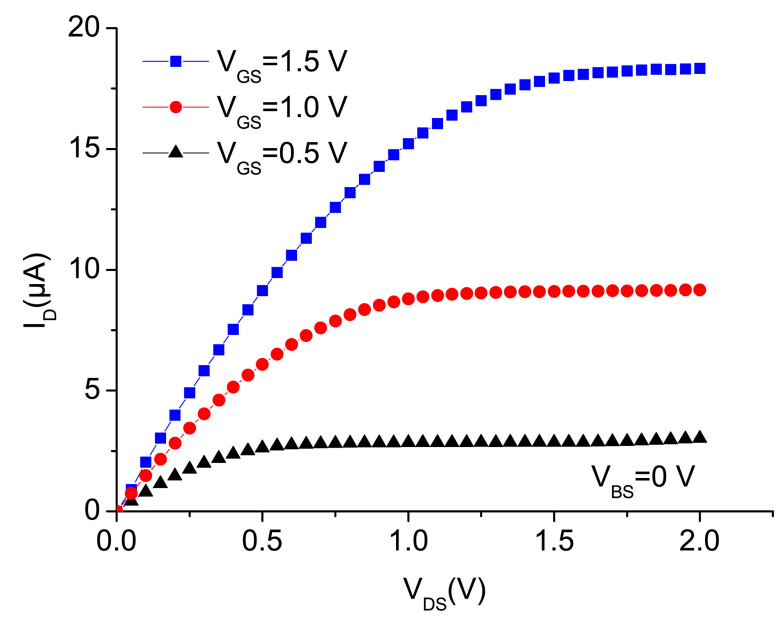

Fig. 6. Output characteristics of the multi-gate dual Si nanowire MOSFET [W0.8, SEM cross-section in Fig. 3 (left)].

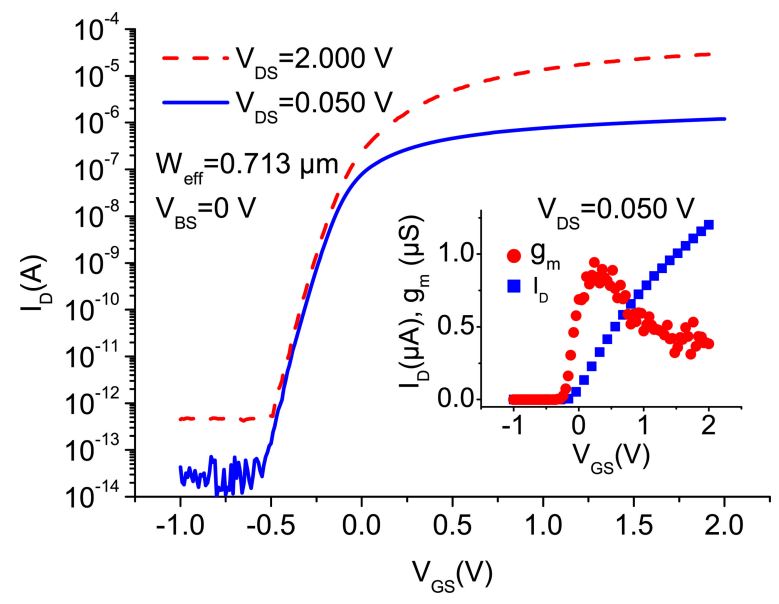

Fig. 7. Input characteristics of the multi-gate dual $\mathrm{Si}$ nanowire MOSFET [W0.8; SEM cross-section in Fig. 3, left; $\mathrm{W}_{\mathrm{eff}}=0.713 \mu \mathrm{m}$ (top NW: $0.273 \mu \mathrm{m}$; bottom NW: $0.440 \mu \mathrm{m})] . \mathrm{SS}=62 \mathrm{mV} /$ decade, $\mathrm{V}_{\mathrm{TH}}=-0.061 \mathrm{~V}$, $\mu_{0}=468 \mathrm{~cm}^{2} / \mathrm{V} \cdot \mathrm{s}$, and $\mathrm{R}_{\mathrm{SD}}=18.7 \mathrm{k} \Omega$, all at $\mathrm{V}_{\mathrm{DS}}=50 \mathrm{mV}$.

MOSFET shows $\mathrm{V}_{\mathrm{TH}}=+0.255 \mathrm{~V}$ and $\mu_{0}=329 \mathrm{~cm}^{2} / \mathrm{V} \cdot \mathrm{s}$ at $\mathrm{V}_{\mathrm{DS}}=50 \mathrm{mV}$. The observed downshift in the threshold voltage of the dual Si nanowire is mainly due to the local volume inversion, corner effect, and uniaxial tensile stress in the channel [26], [27]. The optimum values of the subthreshold slope as well as very low leakage current values are reflecting an excellent gate stack while the narrower channels represent a better value due to a better electrostatic control on the channel. A $42 \%$ low-field electron mobility improvement in the buckled dual Si nanowire in comparison to the omega-gate MOSFET is due to uniaxial tensile stress in the channel. The higher transconductance drop in Fig. 7 in strong inversion regime in comparison to the wider devices is mainly due to the higher series resistance in the scaled Si nanowire MOSFETs.

The series resistance values reported in the caption of Figs. 7-9 are extracted and estimated using the $y$-intercept of total resistance $\left(\mathrm{R}_{\text {tot }}=\mathrm{V}_{\mathrm{DS}} / \mathrm{I}_{D}\right)$ versus $1 /\left(\mathrm{V}_{\mathrm{GS}}-\mathrm{V}_{\mathrm{TH}}-\mathrm{V}_{\mathrm{DS}} / 2\right)$ at $\mathrm{V}_{\mathrm{DS}}=50 \mathrm{mV}$ [28], assuming series resistance as the major transconductance drop mechanism in strong inversion regime.

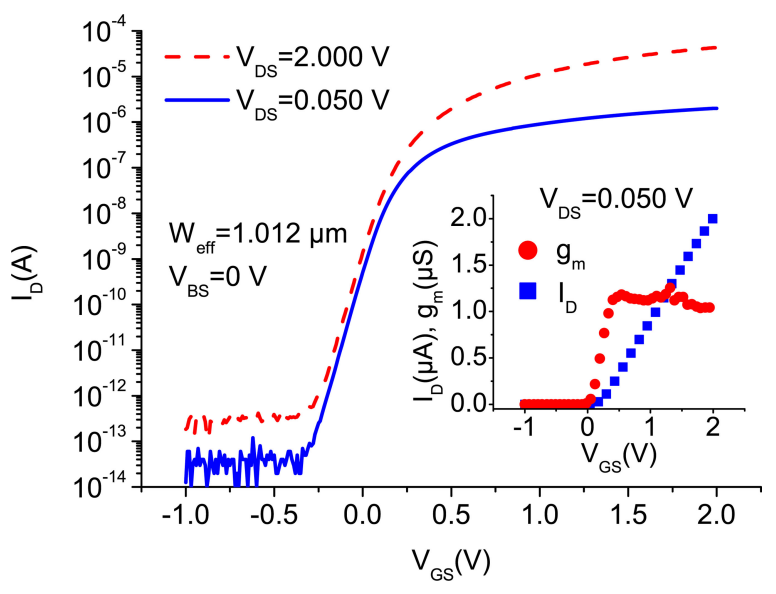

Fig. 8. Input characteristics of the multi-gate Si nanowire MOSFET (W1.0; $\mathrm{SEM}$ cross-section in Fig. 3 (center); $\left.\mathrm{W}_{\text {eff }}=1.012 \mu \mathrm{m}\right) . \mathrm{SS}=64 \mathrm{mV} /$ decade, $\mathrm{V}_{\mathrm{TH}}=0.213 \mathrm{~V}, \mu_{0}=353 \mathrm{~cm}^{2} / \mathrm{V} \cdot \mathrm{s}$, and $\mathrm{R}_{\mathrm{SD}}=3.7 \mathrm{k} \Omega$, all at $\mathrm{V}_{\mathrm{DS}}=50 \mathrm{mV}$

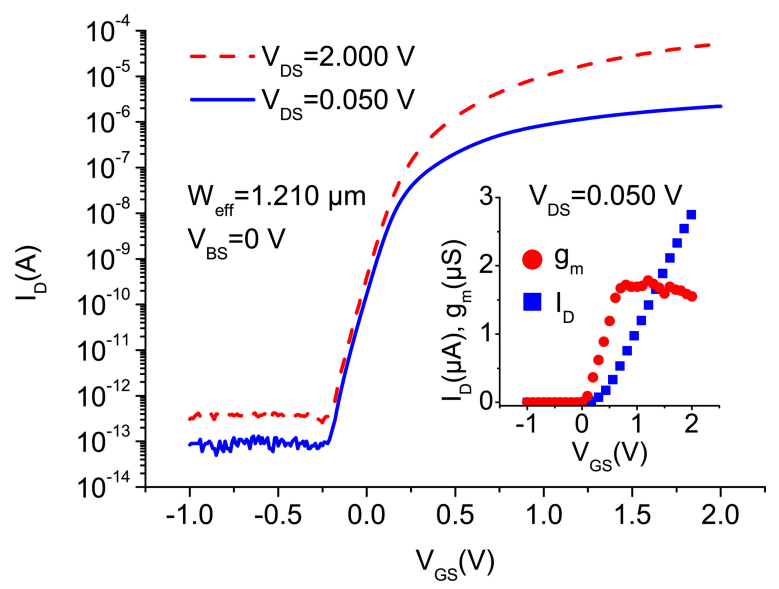

Fig. 9. Input characteristics of the omega-gate MOSFET (W1.2, SEM crosssection in Fig. 3 (right), $\left.\mathrm{W}_{\mathrm{eff}}=1.210 \mu \mathrm{m}\right) . \mathrm{SS}=68 \mathrm{mV} /$ decade, $\mathrm{V}_{\mathrm{TH}}=$ $0.255 \mathrm{~V}, \mu_{0}=329 \mathrm{~cm}^{2} / \mathrm{V} \cdot \mathrm{s}$, and $\mathrm{R}_{\mathrm{SD}}=0.8 \mathrm{k} \Omega$, all at $\mathrm{V}_{\mathrm{DS}}=50 \mathrm{mV}$.

Note that the contribution of intrinsic mobility attenuation factor $\left(\theta_{0}\right.$, see [24], can be influenced mainly by channel-dielectric interface quality [29]) on the transconductance drop in strong inversion should be negligible due to having an excellent gate stack (optimum subthreshold slope and pretty low leakage drain current in a sub-100 fA range for all the multi-gate devices at $\mathrm{V}_{\mathrm{DS}}=50 \mathrm{mV}$ ).

\section{CONCLUSION}

We demonstrated multi-gate buckled self-aligned dual $\mathrm{Si}$ nanowires including two sub-100 nanometer cross-sectional cores on bulk Si substrate using optical lithography, hard mask/spacer technology and local oxidation for the first time. Uniaxial tensile stress was measured in the buckled dual $\mathrm{Si}$ nanowires using micro-Raman spectroscopy and the stressbased low-field electron mobility enhancement was reported. 


\section{ACKNOWLEDGMENT}

The authors would like to thank Dr. P.-E. Hellstrom, KTH Royal Institute of Technology, Stockholm, Sweden, for the gate stack process and Dr. H.M.H. Chong, M.E. Schmidt, and S.M. Sultan, Southampton University, Southampton, U.K., for their technical advice on micro-Raman measurements.

\section{REFERENCES}

[1] T. Kizuka, Y. Takatani, K. Asaka, and R. Yoshizaki, "Measurements of the atomistic mechanics of single crystalline silicon wires of nanometer width," Phys. Review B, vol. 72, p. 035333, 2005.

[2] K. E. Petersen, "Silicon as a mechanical material," Proc. IEEE, vol. 70, pp. $420-457,1982$.

[3] S. Franssila, Introduction to Microfabrication, 2nd ed. New York: Wiley, 2010.

[4] N. Singh, W. W Fang, S. C. Rustagi, K. D. Budharaju, S. H. G. Teo, S. Mohanraj, G. Q. Lo, N. Balasubramanian, and D.-L. Kwong, "Observation of metal-layer stress on Si nanowires in gate-all-around high-k/metal-gate device structures," IEEE Electron. Device Lett., vol. 28, no. 7, pp. 558561, Jul. 2007.

[5] M. Najmzadeh, D. Bouvet, W. Grabinski, and A. M. Ionescu, "Local stressors to accommodate 1.2 to $5.6 \mathrm{GPa}$ uniaxial tensile stress in suspended gate-all-around Si nanowire nMOSFETs by elastic local buckling," IEEE Int. Semicond. Device Res. Symp., pp. 1-2, 2011.

[6] K. Boucart, A. M. Ionescu, and W. Riess, "Asymmetrically strained allsilicon Tunnel FETs featuring $1 \mathrm{~V}$ operation," in Proc. IEEE Eur. SolidState Device Res. Conf., Sep. 2009, pp. 452-456.

[7] M. Najmzadeh, K. Boucart, W. Riess, and A. M. Ionescu, "Asymmetrically strained all-silicon multi-gate n-Tunnel FETs," Solid-State Electron., vol. 54, pp. 935-941, 2010.

[8] P. Hashemi, L. Gomez, M. Canonico, and J. L. Hoyt, "Electron transport in Gate-All-Around uniaxial tensile strained-Si nanowire n-MOSFETs," in Proc. IEEE Int. Electron Devices Meet., 2008, pp. 1-4.

[9] K. E. Moselund, P. Dobrosz, S. Olsen, V. Pott, L. De Michielis, D. Tsamados, D. Bouvet, A. O'Neill, and A. M. Ionescu, "Bended gate-all-around nanowire MOSFET: A device with enhanced carrier mobility due to oxidation-induced tensile stress," in Proc. IEEE Int. Electron Devices Meet., 2007, pp. 191-194.

[10] M. Li, K. H. Yeo, Y. Y. Yeoh, S. D. Suk, K. H. Cho, D.-W. Kim, D. Park, and W.-S. Lee, "Experimental investigation on superior PMOS performance of uniaxial strained $<110>$ silicon nanowire channel by embedded $\mathrm{SiGe}$ source/drain," in Proc. IEEE Int. Electron Devices Meet., 2007, pp. 899902.

[11] B. Liu, H. S. Wong, M. Yang, and Y. C. Yeo, "Strained silicon nanowire p-channel FETs with diamond-like carbon liner stressor," IEEE Electron. Device Lett., vol. 31, pp. 1371-1373, 2010.

[12] M. Najmzadeh, D. Bouvet, W. Grabinski, and A. M. Ionescu, "Uniaxially tensile strained accumulation-mode gate-all-around Si nanowire nMOSFETs," in Proc. IEEE Device Res. Conf., 2011, pp. 145-146.

[13] M. Najmzadeh, D. Bouvet, W. Grabinski, and A. M. Ionescu, "Accumulation-Mode GAA Si NW nFET with sub-5 nm cross-section and high uniaxial tensile strain," in Proc. IEEE Eur. Solid-State Device Res. Conf., 2011, pp. 311-314.

[14] M. Najmzadeh, D. Bouvet, W. Grabinski, J.-M. Sallese, and A. M. Ionescu, "Accumulation-mode gate-all-around Si nanowire nMOSFETs with sub$5 \mathrm{~nm}$ cross-section and high uniaxial tensile strain," Solid-State Electron., vol. 74, pp. 114-120, 2012.
[15] P. C. H. Chan, W. Xusheng, F. Chuguang, C. Mansun, and Z. Shengdong, "Three-dimensional stacked-Fin-CMOS integrated circuit using double layer SOI material," in Proc. IEEE Int. Conf. Solid-State Integrated Circuit Technol., 2004, pp. 81-85.

[16] T. Ernst et al., "Novel 3D integration process for highly scalable NanoBeam stacked-channels GAA (NBG) FinFETs with HfO2/TiN gate stack," in Proc. IEEE Int. Electron Devices Meet., 2006, pp. 1-4.

[17] W. W. Fang, N. Singh, L. K. Bera, H. S. Nguyen, S. C. Rustagi, G. Q. Lo, N Balasubramanian, and D.-L. Kwong, "Vertically stacked SiGe nanowire array channel CMOS transistors," IEEE Electron. Device Lett., vol. 28, no. 3, pp. 211-213, Mar. 2007

[18] K. D. Buddharaju, N. Singh, S. C. Rustagi, S. H. G. Teo, G. Q. Lo, N Balasubramanian, and D.-L. Kwong, "Si-nanowire CMOS inverter logic fabricated using gate-all-around (GAA) devices and top-down approach," Solid-State Electron., vol. 52, no. 9, pp. 1312-1317, 2008.

[19] R. M. Y. Ng, T. Wang, F. Liu, X. Zuo, J. He, and M. Chan, "Vertically stacked silicon nanowire transistors fabricated by inductive plasma etching and stress-limited oxidation," IEEE Electron. Device Lett., vol. 30, no. 5, pp. 520-522, May 2009.

[20] A. L. Theng, W. L. Goh, G. Q. Lo, L. Chan, and C. M. Ng, "Dual nanowire silicon MOSFET with silicon bridge and TaN gate," IEEE Trans. Nanotechnol., vol. 7, no. 6, pp. 795-799, Nov. 2008.

[21] M. Najmzadeh, D. Bouvet, P. Dobrosz, S. Olsen, and A. M. Ionescu, "Investigation of oxidation-induced strain in a top-down Si nanowire platform," Microelectron. Eng., vol. 86, pp. 1961-1964, 2009.

[22] M. Najmzadeh, L. De Michielis, D. Bouvet, P. Dobrosz, S. Olsen, and A M. Ionescu, "Silicon nanowires with lateral uniaxial tensile stress profiles for high electron mobility gate-all-around MOSFETs," Microelectron. Eng., vol. 87, pp. 1561-1565, 2010.

[23] I. D. Wolf, "Micro-Raman spectroscopy to study local mechanical stress in silicon integrated circuits," Semicond. Sci. Technol., vol. 11, pp. 139-154, 1996.

[24] G. Ghibaudo, "New method for the extraction of MOSFET parameters," Electron. Lett., vol. 24, pp. 543-545, 1988.

[25] G. Ghibaudo, "Critical MOSFETs operation for low voltage/low power ICs: Ideal characteristics, parameter extraction, electrical noise and RTS fluctuations," Microelectron. Eng., vol. 39, pp. 31-57, 1997.

[26] J. S. Lim, S. E. Thompson, and J. G. Fossum, "Comparison of thresholdvoltage shifts for uniaxial and biaxial tensile-stressed n-MOSFETs," IEEE Electron. Device Lett., vol. 25, no. 11, pp. 731-733, Nov. 2004.

[27] J. G. Fossum, J.-W. Yang, and V. P. Trivedi, "Suppression of corner effects in triple-gate MOSFETs," IEEE Electron. Device Lett., vol. 24, no. 12, pp. 745-747, Dec. 2003.

[28] P. Hashemi, L. Gomez, and J. L. Hoyt, "Gate-All-Around n-MOSFETs with uniaxial tensile strain-induced performance enhancement scalable to sub-10-nm nanowire diameter," IEEE Electron. Device Lett., vol. 30, no. 4, pp. 401-403, Apr. 2009.

[29] K. E. Moselund, M. Najmzadeh, P. Dobrosz, S. H. Olsen, D. Bouvet, L. De Michielis, V. Pott, and A. M. Ionescu, "The high-mobility bended n-channel silicon nanowire transistor," IEEE Trans. Electron. Devices, vol. 57, no. 4, pp. 866-876, Apr. 2010.

Authors' photographs and biographies not available at the time of publication. 